\title{
VERSATILIDADE DO RETALHO MUSCULOCUTÂNEO DOTENSOR DA FÁSCIA LATA
}

VERSATILITY OF THE FASCIA LATA TENSOR MUSCULOCUTANEOUS FLAP

Rogério dos Santos Ramos, TCBC-BA'; Marcelo Sacramento Cunha, TCBC-BA²; Rodolfo Oliveira de Jesus ${ }^{3}$; Igor de Almeida Basílio ${ }^{3}$; Adson Figuerêdo ${ }^{4}$; Luiza Cavalcanti Fadul ${ }^{3}$

\begin{abstract}
RESUMO: Objetivo: Relatar a experiência com a utilização do retalho musculocutâneo do tensor da fáscia lata (TFL em diferentes situações clínicas, evidenciando suas diversas aplicações, analisando e discutindo as indicações, resultados e complicações. Método: Entre janeiro de 2003 e dezembro de 2005 dezessete retalhos miocutâneos do TFL foram realizados para cobertura cutânea em uma variedade de defeitos em quinze pacientes.Durante o ato operatório a equipe optou pelo posicionamento do paciente em decúbito lateral em oposição ao lado da lesão a ser reparada. Resultados: Houve sucesso com cobertura cutânea adequada em todos os casos. Em quatro destes ocorreu necessidade de enxerto de pele parcial na área doadora. Em dois casos houve isquemia distal do retalho e em um aconteceu pequena deiscência de sutura em zona doadora. Conclusão: O retalho miocutâneo do tensor da fáscia lata, portanto, possibilita uma excelente cobertura cutânea para tratamento de defeitos em diversos segmentos anatômicos com pouca morbidade em área doadora (Rev. Col. Bras. Cir. 2008; 35(3): 155-158).
\end{abstract}

Descritores: Retalhos Cirúrgicos/utilização; Músculos/transplante; Fáscia Lata/cirurgia.

\section{INTRODUÇÃO}

O retalho do Tensor da Fáscia Lata (TFL) tem sido a primeira opção para o tratamento das úlceras de pressão trocantéricas desde o final da década de 70. Além disso, possui grande versatilidade, podendo ser utilizado em inúmeras situações. A grande quantidade de pele disponível e segurança do pedículo são as suas principais características, além da porção cutânea que pode ser até três vezes maior que as dimensões do músculo. O TFL pode ser utilizado desde um retalho muscular até osteomiocutâneo, sendo geralmente musculocutâneo. Ele permite também a confecção de retalhos com dois possíveis arcos de rotação: um anterior, cobrindo a região inguinal, perineal, pubiana, hipogástrica e a região abdominal superior, ou um arco posterior, que pode cobrir a área trocantérica, isquiática, perianal e sacral. Além disso, a zona doadora pode ser fechada primariamente a depender das dimensões do retalho. Dentre as inúmeras aplicações do TFL estão a cobertura das regiões: abdominal, perineal, trocantérica, isquiática e sacral; a reconstrução de parede abdominal, vulva e hérnias inguinais, e o transplante microcirúrgico para reconstrução de cabeça e pescoço, extremidades superiores e inferiores, além da reconstrução mamária.

Os autores relatam sua experiência com a utilização do retalho musculocutâneo do TFL em diversas situações, sendo analisados e discutidos as indicações, os resultados e as complicações.

\section{MÉTODO}

Entre janeiro de 2003 e dezembro de 2005 foram realizados dezessete retalhos miocutâneos do Tensor da Fáscia Lata para cobertura cutânea em uma variedade de defeitos (Figura 1). Quinze pacientes participaram do estudo. Dentre estes, oito retalhos foram realizados para tratamento de úlce-

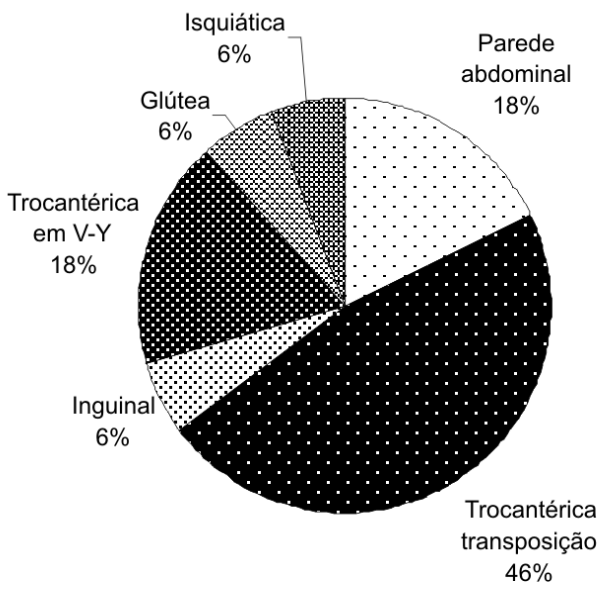

$\begin{array}{ll}\because \text { Parede abdominal } & \text { - Trocantérica transposição } \\ \because \text { Inguinal } & \text { a Trocantérica em V-Y } \\ \approx \text { Glútea } & \text { \& Isquiática }\end{array}$

Figura 1 - Distribuição dos casos por locais de reconstrução.

\footnotetext{
1. Professor Substituto da Disciplina de Cirurgia Plástica da FAMED (UFBA); Professor Doutor da Disciplina de Cirurgia Plástica da FAMED (UFBA);

Membro Titular da Sociedade Brasileira de Cirurgia Plástica.

2. Médico Assistente da Disciplina de Cirurgia Plástica da FAMED (UFBA); Membro Titular da Sociedade Brasileira de Cirurgia Plástica.

3. Acadêmico de Medicina e Membro da Liga Baiana de Cirurgia Plástica.

4. Cirurgião Geral e Membro Colaborador da Liga Baiana de Cirurgia Plástica

Recebido em 21/11/2007

Aceito para publicação em 21/01/2008

Conflito de interesses: nenhum

Fonte de financiamento: nenhuma

Trabalho realizado na Disciplina de Cirurgia Plástica do Departamento de Cirurgia da Universidade Federal da Bahia.
} 
ras de pressão trocantéricas sob a forma de transposição, três com o mesmo objetivo, porém na forma de V-Y. Foram realizados ainda três retalhos para reconstrução da parede abdominal (Figuras 2 e 3), um para tratamento de úlcera de pressão isquiática recidivada, um para a região inguinal e um retalho para reconstrução glútea após extensa ressecção oncológica (Figura 4 e 5). Durante o ato operatório a equipe optou pelo posicionamento do paciente em decúbito lateral em oposição ao lado da lesão a ser reparada.

\section{RESULTADOS}

A confecção deste retalho promoveu a cobertura cutânea adequada em todos os pacientes. Não houve perda total do retalho. Em quatro pacientes (24\%) houve necessidade de enxerto de pele parcial em área doadora dos retalhos. Em dois pacientes $(13 \%)$ houve isquemia distal do retalho com necrose epidérmica (Figura 6). Apenas em um paciente houve pequena deiscência de sutura em zona doadora, porém sem comprometimento do resultado final.

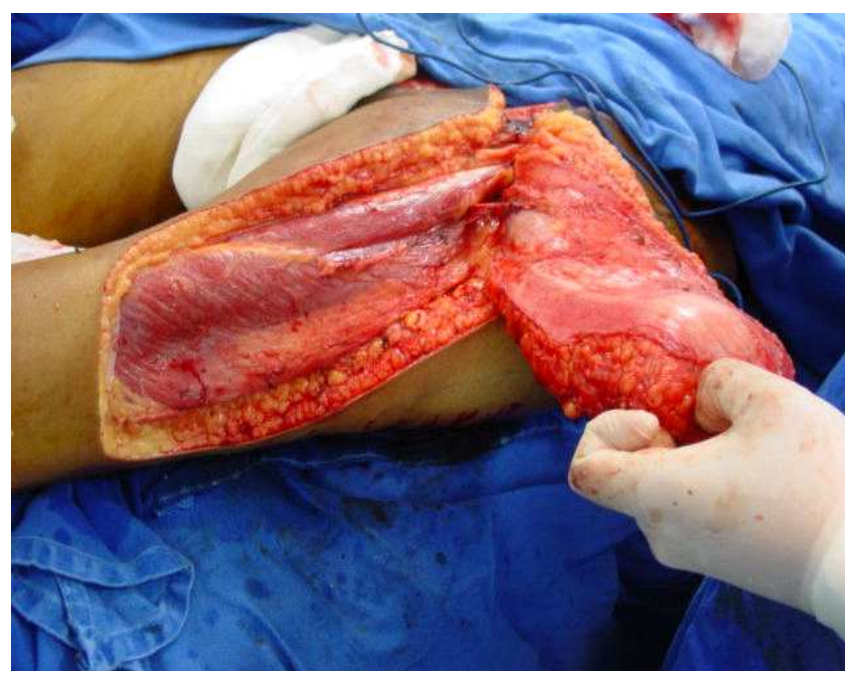

Figura 2 - Retalho pediculado do TFL para reconstrução parede abdominal.

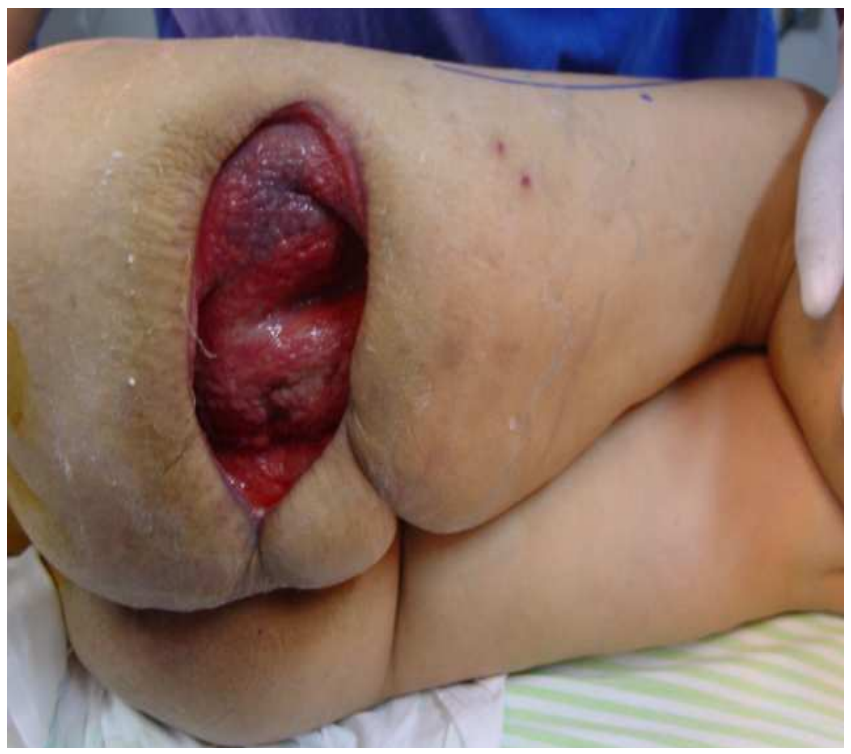

Figura 4 - Defeito região glútea após ressecção oncológica.

\section{DISCUSSÃO}

O papel do retalho do tensor da fáscia lata é bem estabelecido na literatura e a sua introdução possibilitou um grande avanço no tratamento de diversos tipos de defeitos, inclusive aqueles de espessura total em diversos segmentos corporais. Isso decorre do fato de que a fáscia e o tecido músculo-cutâneo do TFL fornecem uma estrutura tal que complementa a reconstrução dessas lesões ${ }^{1}$. Em vista disso, seu uso é descrito em inúmeros estudos, que também demonstram baixo índice de complicações e necessidade de revisões cirúrgicas. Percebe-se, portanto, que algumas características peculiares são de fundamental importância para garantir essa aplicabilidade.

Este retalho possui vascularização do tipo I e seu pedículo baseia-se na artéria femoral circunflexa lateral, que emite perfurantes músculo-cutâneas para o TFL ${ }^{2-4}$. Além desse pedículo vascular ser longo $(5-6 \mathrm{~cm})$, bem definido e possuir uma posição constante, permitindo uma dissecção habitualmente simples, ele também possui um diâmetro externo

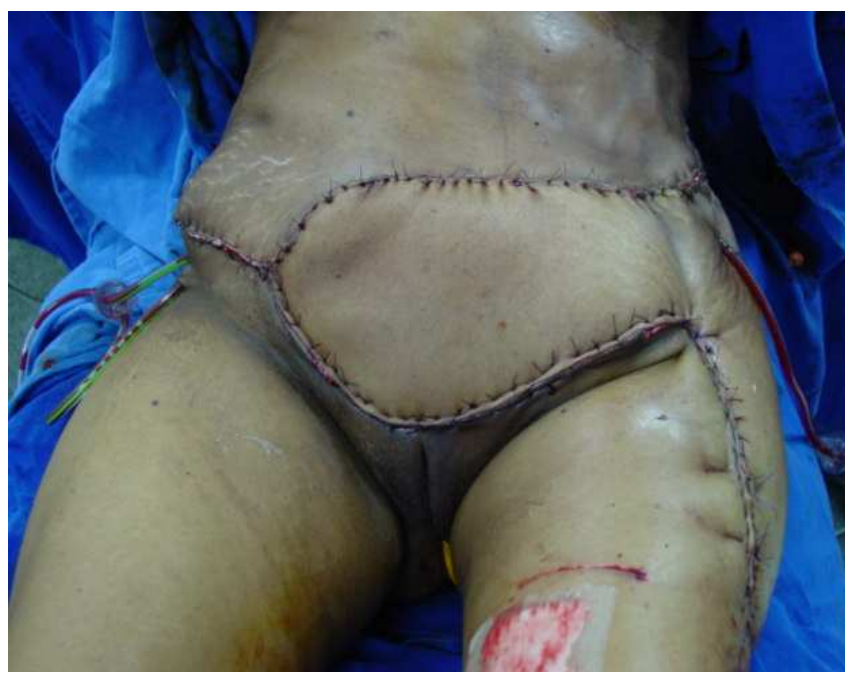

Figura 3 - Retalho posicionado no abdome.

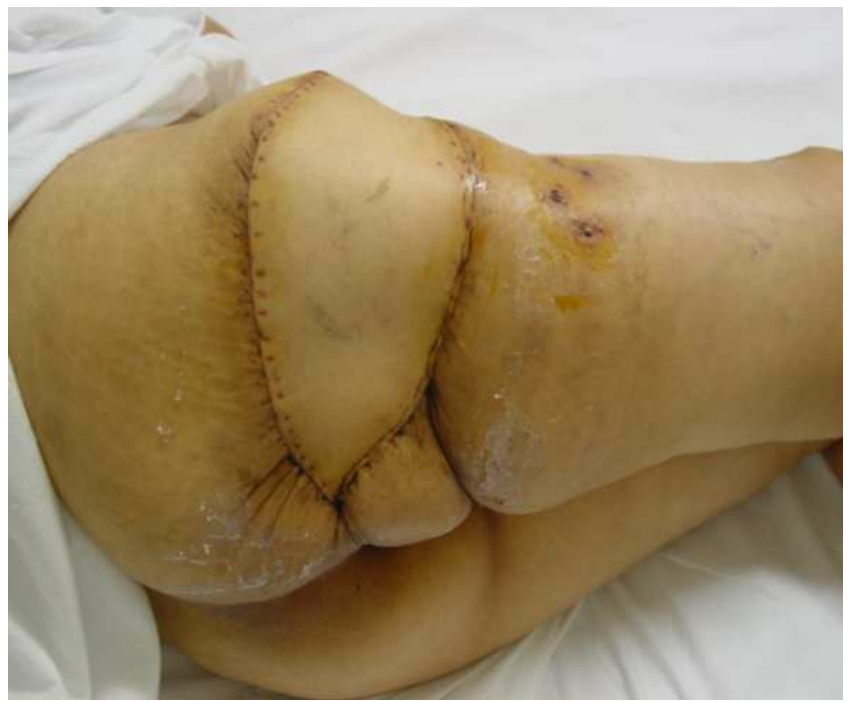

Figura 5 - Pós-operatório recente após transposição do retalho miocutâneo do TFL. 

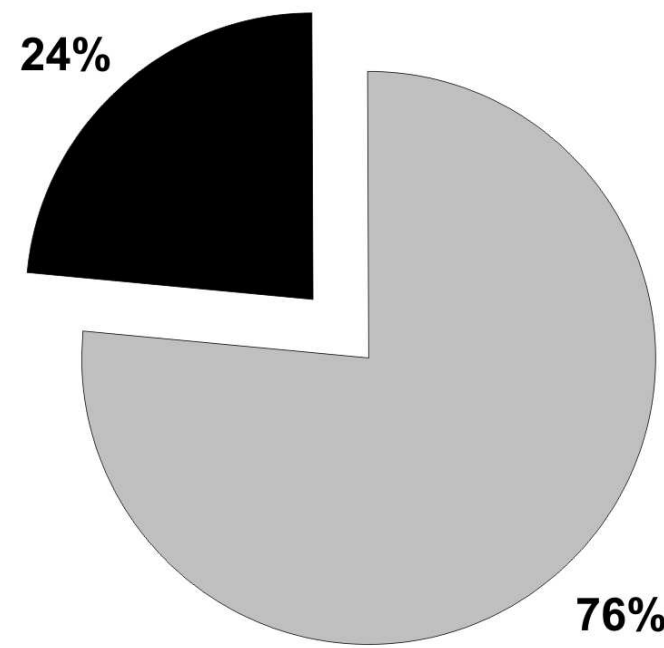

$\square$ Fechamento primário $\square$ Enxerto de pele parcial

Figura 6 - Opções para síntese de área doadora do retalho.

apropriado (1.5-2 mm) para transferência microcirúrgica. Já o território vascular cutâneo consistente e extenso está associado às perfurantes em grande quantidade, que por sua vez, garantem a viabilidade e segurança do retalho ${ }^{2,5}$.

O músculo TFL, que compõe esse retalho, situa-se na porção lateral da perna. Tem origem na espinha ilíaca ânterosuperior e inserção junto às fibras da fáscia lata, ao nível do côndilo lateral da tíbia. Ele é capaz de proporcionar a confecção de um retalho miocutâneo de fácil execução e tem como importante vantagem proporcionar um retalho completo com um grande arco de rotação. Essa característica permite reconstruções em variados ângulos, podendo alcançar até o terço superior da parede abdominal, ou ter acesso à área inguinal, local de difícil reconstituição por outras técnicas ${ }^{2,6}$.

Além disso, esse retalho fornece tecido em grande quantidade, principalmente a porção cutânea, e uma forte sustentação fascial, eliminando, conseqüentemente, a necessidade de próteses sintéticas, principalmente nas reconstruções da parede abdominal ${ }^{1,5,7}$. Esses tecidos, cutâneo e subcutâneo, têm ainda tipicamente uma cor muito próxima à maioria das áreas lesionadas que são reconstruídas, fornecendo, desse modo, uma cobertura estética externa aceitável ${ }^{7}$. A estrutura anatômica do TFL possibilita assim a sua utilização tanto como um retalho pediculado quanto microcirúrgico, O primeiro tem sido escolha para reparo em lesões de tamanho limitado, mas que requerem fáscia e tecido conjuntivo ${ }^{1,6}$. Entretanto, quando baseado no pedículo vascular dominante o arco da rotação limita o uso deste retalho à correção de alguns defeitos mais baixos da parede abdominal ${ }^{5}$. O uso deste tipo de retalho em posições mais distantes parece estar relacionado com maiores índices de complicações, que possivelmente estão relacionadas ao uso do terço distal menos vascularizado. A utilização do retalho livre, por outro lado, possibilita a eliminação do arco de rotação, além de uma redução maior de morbidade em área doadora ${ }^{1,5,7}$. Ele é preferível em defeitos supra-umbilicais ou quando houver necessidade de usar dois retalhos pediculados para a cobertura do defeito, eliminando ainda a necessidade de incluir o terço distal do retalho. Esta porção de tecido está mais susceptível ás alterações isquêmicas ${ }^{1,5,7}$. Este tipo de retalho propicia as seguintes vantagens adicionais que pode ser dissecado com o paciente na posição supina, é capaz de suportar doses tumoricidas da radioterapia, e a perda do músculo na área doadora não resulta em um déficit funcional significativo na flexão ou abdução do quadril ${ }^{5}$. Por outro lado, também apresenta algumas desvantagens tais como: maior tempo operatório, potencial perda de retalho, e a necessidade de utilização de técnicas microcirúrgicas ${ }^{1}$.

Devido às características e variedade de técnicas inerentes a ele, o retalho do TFL possui uma versatilidade para reconstrução que tem sido demonstrada em numerosas especialidades cirúrgicas. Em cirurgia plástica tem sido usado mais comumente para o reparo de ulceras de pressão e reconstrução de parede tóraco-abdominal após excisão de tumor, mas também pode ser usado para reconstrução de faringe e esôfago, reparo de palato, reanimação e "lifting facial"1.

Contudo, esse retalho não está isento de complicações. Algumas delas não influenciam no resultado final e necessitam de reoperação pouquíssimas vezes. As taxas de complicações na área de reconstrução podem chegar até a 48\%, enquanto que na zona doadora podem alcançar 18,5\% ${ }^{1}$.

As revisões realizadas por $\mathrm{O}^{\prime}$ Hare e Williams demonstraram, respectivamente, que de três pacientes que utilizaram retalhos pediculados estendidos (alcançando $5 \mathrm{~cm}$ do joelho) para correção de grandes defeitos na parede abdominal, dois deles necessitaram de reabordagem devido à necrose de pele na ponta do retalho e que de nove pacientes submetidos a reconstrução abdominal com retalho pediculado, um também necessitou de semelhante reabordagem ${ }^{1,8}$. Este achado parece ser similar a nossa experiência onde houve isquemia e necrose distal do retalho em apenas dois pacientes. Por outro lado, em uma revisão feita por Nahai, com 54 casos de reconstrução com este tipo de retalho, não foi relatada complicação alguma ${ }^{9}$.

A relativa morbidade em área doadora encontrada no presente estudo, onde houve necessidade de enxerto de pele parcial em quatro pacientes, parece estar relacionada às grandes dimensões dos retalhos. Alguns autores afirmam que há maior morbidade quando existe a necessidade de confecção de mais que $8 \mathrm{~cm}$ de retalho, o que normalmente requer um enxerto para síntese da área doadora ${ }^{6}$.

Para superar as desvantagens da técnica de transposição posterior algumas modificações foram desenvolvidas, como o avanço em V-Y e a posição retrógrada com V-Y, que superam a deformidade em orelha de cachorro, além de colocar a parcela mais grossa do retalho sobre o defeito ós$\mathrm{seo}^{4}$. O uso concomitante do retalho do músculo glúteo médio possibilita uma reutilização do $\mathrm{TFL}^{4}$. E o uso do retalho musculocutâneo do TFL bilobado supera o problema da junção em T entre o_ponto da rotação e a área doadora, que é geralmente fechada sob tensão ${ }^{4}$. De acordo com Aslan, essa excessiva tensão e eventual separação da sutura na confluên- 
cia da área doadora com o retalho é o problema mais comum envolvendo o uso do $\mathrm{TFL}^{10}$, evento que houve em um dos nossos pacientes, porém de forma limitada, exigindo uma sutura simples para correção. Contudo, avanços ainda maiores têm sido alcançados através de alterações na confecção desse retalho, como a modificações propostas por Demirseren $e t$ al em 2003 e por Aslan et al em 2005 que auxiliam a resolver os problemas da clássica transposição posterior com segurança e praticidade ${ }^{4,10}$.

A despeito das complicações observadas, foi obtido sucesso na cobertura cutânea em todos os casos. Essa eficá- cia do TFL em restaurar a integridade da área lesada, também relatada na literatura, está possivelmente relacionada com a adequada seleção da forma apropriada de retalho.

O retalho miocutâneo do tensor da fáscia lata, portanto, possibilita uma excelente cobertura cutânea para tratamento de defeitos em diversos segmentos anatômicos com pouca morbidade em área doadora. Trata-se de um retalho extremamente seguro, versátil, de fácil e rápida execução, apresentando relativamente poucas complicações, podendo ser utilizado em diversas situações clínicas com sucesso.

\begin{abstract}
Background: The fascia lata tensor (FLT) musculocutaneous flap holds a great versatility. It is used in countless situations. We present our experience with the use of this flap in several circumstances. We also analyze and discuss about indications, results and complications. Methods: Between January 2003 and December 2005, 15 patients (10 man and 5 women) underwent 17 FLT flaps to treat many skin defects. Patients were between 18 and 65 years-old. Patients' position during surgery was lateral decubitus. Follow up has been obtained from 6 months to 3 years. Results: We succeed with fit skin coverage in all cases. Partial skin graft was necessary in four cases. Distal flap ischemia occurred in two patients. Small dehiscence at the donor site was seen (and treated by simple suture) in one case. Conclusion: The FLT musculocutaneous flap is extremely reliable and versatile; it has easy and fast execution and relatively few complications. It can successfully be used in many clinical situations.
\end{abstract}

Key words: Surgical flaps/utilization; Muscles/transplantation; Fascia Lata/surgery.

\section{REFERÊNCIAS}

1. Williams JK, Carlson GW, deChalain T, Howell R, Coleman JJ. Role of tensor fasciae latae in abdominal wall reconstruction. Plast Reconstr Surg. 1998; 101(3):713-8.

2. Yamaguchi C, Souen J. Cirurgia de reconstrução de vulva. Rev Soc Bras Cancerol [periódico na Internet]. 2000 [acesso em 2006 Dec 20]; (11):[aproximadamente 8 p.]. Disponível em: http:// www.rsbcancer.com.br/rsbc/11 artigo1.asp?nrev $=\mathrm{N}^{\circ} 11$

3. Figueiredo JCA, Freitas AG, Arantes HL. Retalhos musculares e musculocutâneos. In: Mélega JM, Bastos JAV, Mélega LM. Cirurgia plástica, fundamentos e arte, princípios gerais. Rio de Janeiro: MEDSi; 2002. p.121-39.

4. Demirseren ME, Gokrem S, Ozdemir OM, Katircioðlu A, Can Z, Serel S. Hatchet-shaped tensor fascia lata musculocutaneous flap for the coverage of trochanteric pressure sores: a new modification. Ann Plast Surg. 2003; 51(4):419-22.

5. Lyle WG, Gibbs M, Howdieshell TR. The tensor fascia lata free flap in staged abdominal wall reconstruction after traumatic evisceration. J Trauma. 1999; 46(3):519-22.

6. Rohrich RJ, Lowe JB, Hackney FL, Bowman JL, Hobar PC. An algorithm for abdominal wall reconstruction. Plast Reconstr Surg. 2000;105(1):202-16; quiz 217.

7. Chevray PM, Singh NK. Abdominal wall reconstruction with the free tensor fascia lata musculofasciocutaneous flap using intraperitoneal gastroepiploic recipient vessels. Ann Plastic Surg. 2003; 51(1):97-102.

8. O'Hare PM, Leonard AG. Reconstruction of major wall defects using the tensor fasciae latae myocutaneous flap. Br J Plast. Surg. 1982; 35(3):361-6

9. Mathes SJ, Nahai F. Reconstructive surgery principles, anatomy and technique. 1ST ed. New York: Churchill Livingstone; 1997.

10. Aslan G, Tuncali D, Bingul F, Ates L, Yavuz N. The "duck" modification of the tensor fascia lata flap. Ann Plast Surg. 2005; 54(6):637-9.

Como citar este artigo:

Ramos RG, Cunha MS, Jesus RO, Basílio IA, Figuerêdo A, Fadul LC. Versatilidade do retalho musculocutâneo do tensor da fáscia lata. Rev Col Bras Cir. [periódico na Internet] 2008; 35(3). Disponível em URL: http://www.scielo.br/rcbc

Endereço para correspondência

Rogério dos Santos Ramos

Rua Professor Sabino Silva 749/apto 402

Jardim Apipema

40155-250 - Salvador - BA

Email-rog.ramos@uol.com.br

Telefones: (71) 3245-3221 / 9192-1777 Fax: (71) 3245-3221 